\title{
ARCGIS DATA MODELS FOR MANAGING AND PROCESING IMAGERY
}

\author{
Hong Xu, Peter Becker \\ Environment System Research Institute (Esri) \\ Redlands, California, USA \\ hxu@esri.com,pbecker@esri.com
}

KEY WORDS: Imagery, processing, GIS, mosaic, cataloging

\begin{abstract}
:
As remote sensing technology advances, imagery becomes one of the most important data sources for GIS applications-as it can quickly provide the most current and most detailed information with high resolution image data. Traditional image processing workflow becomes inefficient in GIS applications especially in the case of disaster analysis. This paper introduces two ArcGIS data models that are designed for on-demand image processing in managing imagery: raster product and mosaic dataset. A raster product allows you to access single scene remote sensing image products dynamically such as a pan-sharpened and orthorectified GeoEye image or multi-spectral Landsat scene with a single drag and drop to the map display. Mosaic datasets allow you to catalog a large collection of images from many sensor platforms, process them on-the-fly and dynamically mosaic the images together. The key concept-the raster function that is used to implement on-the-fly image processing capabilities-will also be discussed.
\end{abstract}

\section{INTRODUCTION}

As one of the most important data sources for Geographic Information Systems (GIS), imagery has been widely used in many GIS applications such as mapping, urban planning, environmental research, disaster analysis, and so on. In the past, remote sensing images had to be preprocessed using remote sensing or photogrammetric software packages before their use in GIS applications. This process normally involved orthorectification, pan-sharpening, image enhancing, mosaicking, clipping, etc. Each process generated many intermediate results which took up a lot of disk space, and the space multiplied if multiple products were required. Also, the time taken to write intermediate results to the disk prolonged the whole processing time, especially for GIS systems or projects that involved large amount of image data. The traditional workflow became inefficient in updating GIS systems and could not meet the requirement of the rapid response system for disaster analysis. As image volumes and resolutions increase, GIS systems must be able to process remote sensing images faster, manage images more efficiently, and deliver the final products to users in a timely fashion. To meet these requirements, recently released ArcGIS software packages have introduced two data models-the raster product and mosaic dataset - that are used for on-the-fly access of remote sensing image products, from either a single image scene or a mosaic of multiple image scenes. These provide a solution for GIS users that need to quickly access and use remote sensing images in their GIS applications.

\section{ARCGIS DATA MODELS}

ArcGIS software can read not only the processed images, e.g. georeferenced and orthorectified images stored in any image format, but also the preprocessed images from various sensor platforms. The preprocessed imagery contains image data and the associated metadata such as band wavelength, sensor camera model, sun angle, sun azimuth, cloud coverage and other information which will be used in processing the image data and producing image products that can be used in GIS applications. These include multiple-band natural color products (RGB), false color infrared products (RGBI), pan-sharpened products, and so on. The raster product model allows users to access these products from a single image scene with an easy drag and drop operation, while the mosaic dataset model allows the user to access a mosaic of these products from multiple image scenes by performing onthe-fly image processing and dynamic mosaicking. Furthermore, the mosaic dataset model provides image cataloging capabilities which allow image searching and discovery through the web. The on-the-fly processing capabilities of the two models are based on a concept called raster function which defines the process chain that will be applied to process the image data.

\subsection{Raster Functions}

A raster function is a pixel based mathematical model that defines an image processing operation. A raster function contains arguments which include the input image and other parameters required by the function. For example, a hillshade function contains a reference to a digital elevation model (DEM) file, an azimuth parameter, a latitude parameter, and a $\mathrm{z}$ factor parameter. When the image is accessed, the rendered pixels in the display will be hillshaded. ArcGIS supports many raster functions, including functions that perform geometric processing (polynomial transformation, RPC transformation, and frame camera transforms), radiometric processing (various stretch algorithms, pan-sharpening, and filtering), image processing (clipping and mosaicking), and terrain processing (hillshade, slope, and aspect). Furthermore, ArcGIS software provides capabilities that allow users to augment existing processing capabilities by adding a custom raster function if it is not supported out of the box. 
A unique characteristic of a raster function is that it stores process definitions with the data instead of storing processed pixels. Upon request, the function processes the pixels at the required resolution and extent-not necessarily the whole image. This reduces the processing time by avoiding processing pixels that are not needed and avoiding writing pixels to disk unnecessarily. Figure 1 is an example of a hillshade function that is applied to a TIFF file containing DEM data. You can simply change the azimuth parameter of the function dynamically to produce different display results.

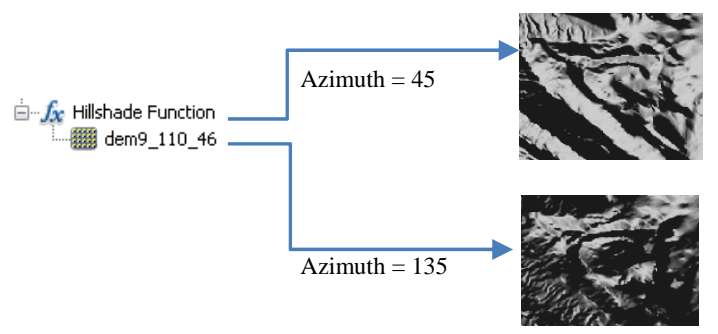

Figure 1. A dynamic display of hillshade at azimuth of 45 and 135 from a DEM file

Multiple raster functions can be chained together to create a composite function. The advantage of composite functions is that one does not need to output intermediate results; instead they can produce the final image directly and quickly. The example below contains a Composite Band function which composes a multispectral image from bands stored in separate TIFF files, a stretch function, and then a clip function that clips a portion of the image based on an area of interest. All the processes are being done onthe-fly without intermediate results being created.

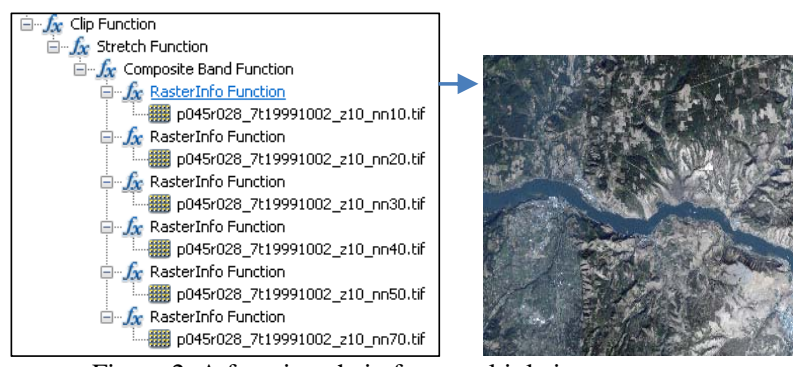

Figure 2. A function chain from multiple images to create a RGB display of an area of interest

The use of the raster function reduces the disk space usage and time taken to process the image by avoiding writing the intermediate results on disk and by processing only the requested pixels. The raster function provides a foundation for dynamic image processing and it is being used in two data models in managing and using remote sensing images.

\subsection{Raster Product}

A raster product is a data model for accessing the image products of single image scene. It is the implementation of the raster function concept to the supported sensor platforms. When you browse the image metadata file from ArcGIS's Catalog window, the system will automatically construct a raster function chain based on sensor specific metadata information from the image scene and produce virtual views of the raster products. In this example of a Landsat 7 ETM+ image scene, ArcGIS's Catalog window allows you to browse the raster product from the .met file and directly access the multi-spectral product, the panchromatic product, the pan-sharpened product, and the thermal product of that image scene (Figure 3). Simply drag and drop any product to ArcMap, and you will get a fully processed raster product that is ready to use. The function chain (Figure 4) shows the image processes that are performed behind the drag-and-drop operation.

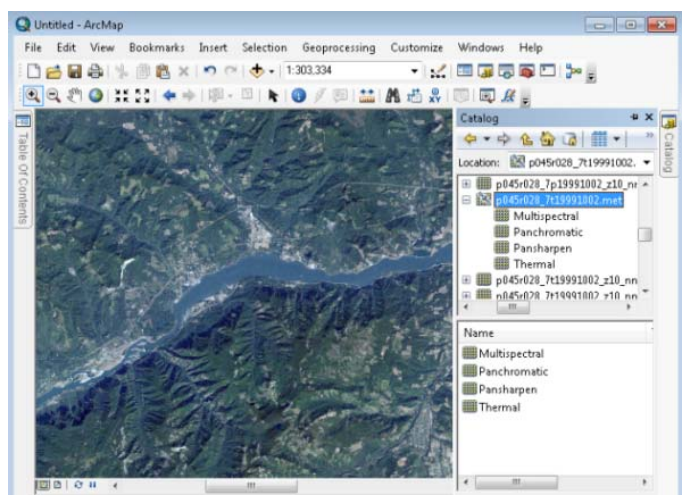

Figure 3. Raster products from a Landsat 7 ETM+ scene

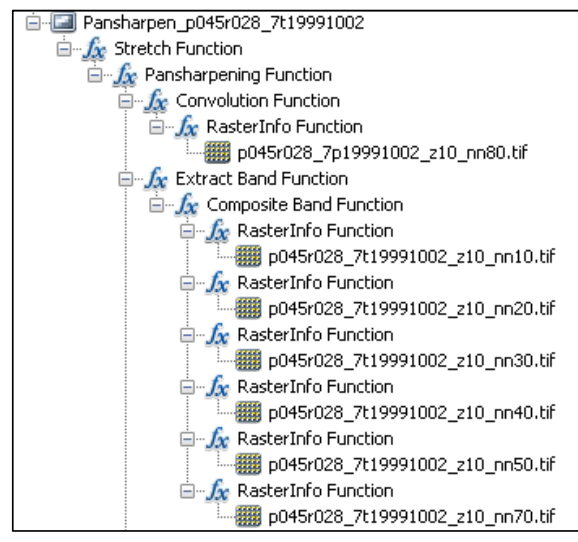

Figure $4 \mathrm{~A}$ raster function chain of the Pansharpen product

The raster product data model shields the complicated image processing steps and provides users with direct access to remote sensing image products that can be used in GIS applications. ArcGIS supports raster products for most commercial satellites such as Landsat, QuickBird, IKONOS, GeoEye, WorldView, and so on. For a complete list of supported products, visit the ArcGIS product Help system at http://resources.arcgis.com/.

\subsection{Mosaic Dataset}

The mosaic dataset data model is designed for handling multiple image scenes. It supports data from many sensor platforms. The mosaic dataset can be used to catalog a large image collection and virtually mosaic them into a seamless image mosaic. Furthermore, mosaicking and cataloging capabilities of the mosaic dataset can 
be accessed by web users using ArcGIS Server, an ArcGIS product that publishes GIS resources as web services.

\subsubsection{Storage scheme}

A mosaic dataset manages images using a set of internal components in the geodatabase (the geodatabase is a way of managing geographic datasets in file system or database within ArcGIS):

1) A footprint table contains a geometric field for storing the polygons of the image footprints, a raster field stores raster values that are composed from references to the image data, along with the raster functions which will be used to process the image data on-the-fly, the MinPS and a MaxPS fields define the visible ranges of the rasters, and many other fields are automatically populated based on metadata information when images are added to the mosaic dataset-such as sensor names, acquisition data, cloud cover, and so on. (Figure 5)
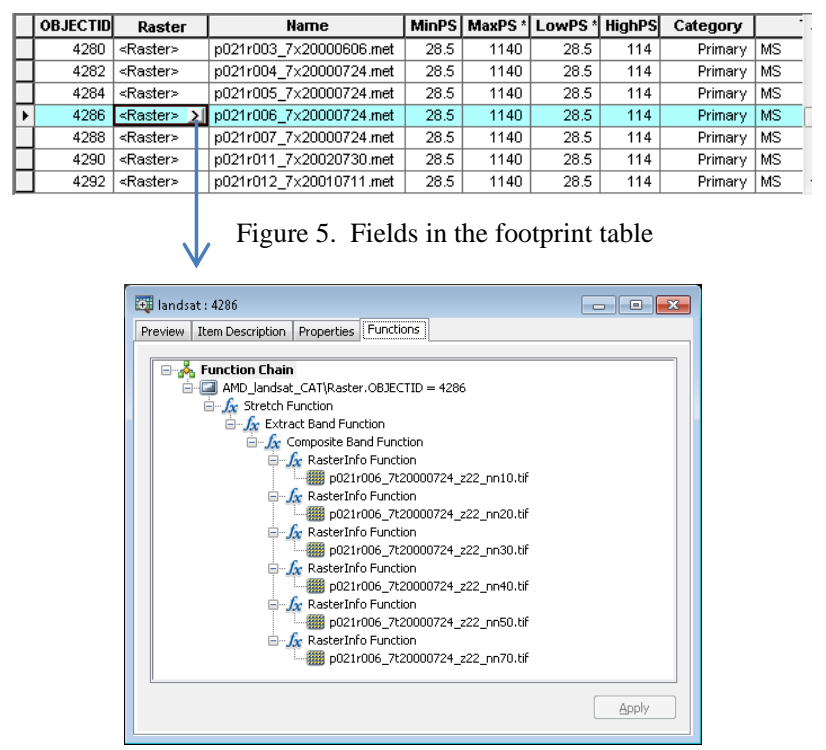

Figure 6: Raster function chain used to create the raster value in the mosaic dataset

2) A boundary table defines the boundary of the image collection. The boundary is computed by the union of the image footprints; however, it can be edited if certain pixels need to be excluded from the display.

3) A raster type table stores raster type instances that are used when crawling and adding data to the mosaic dataset. A raster type is a template that defines raster properties and processes based on sensor platform specification. It is used to construct the raster when adding data to the mosaic dataset. (Figure 6)

4) If calculated, a mosaic dataset may contain a seamline table which is used to store the seamline features for seamline mosaicking.

5) If calculated, a mosaic dataset may also contain a color correction table which stores the image statics for color correction.
6) A mosaic dataset contains many other components such as a log table which stores the operations that have been performed on the mosaic dataset, mosaic rules which are used to define the sorting order for visualizing the rasters in the mosaic dataset, and many properties for accessing and display the mosaic dataset.

\subsubsection{Display mechanism}

A mosaic dataset is displayed as a composite layer of Footprint, Boundary, and Image which provides a virtual mosaic view of the rasters within the mosaic dataset (Figure 7). It can also contain a seamline layer if it is computed. During zooming and panning, the rasters that meet the given extent and visible range request will be selected and sorted based on the user specified mosaic rule and create a virtual seamless mosaic on the fly. For example, you can visualize the mosaic dataset using the Closest To Center mosaic method, which will sort the rasters based on the distance to the center of the display, or by the Seamline method which will use the seamline features to mosaic the selected rasters.

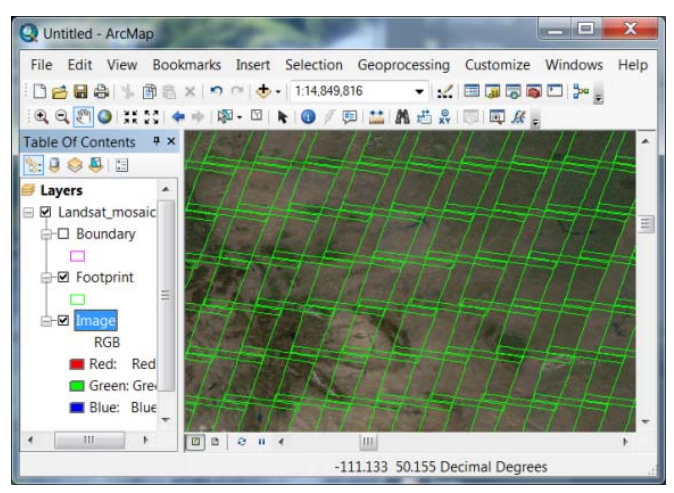

Figure 7. Catalog and mosaic view of a mosaic dataset created from Landsat 7 ETM+ scenes

\subsubsection{Use of mosaic dataset}

The mosaic dataset can be used in many applications including creating ortho image mosaics, creating ortho image tiles, image cataloging, and searching.

1) Create ortho image mosaics or ortho image tiles: The mosaic dataset supports on-demand image processing. You can add images from different sensor platforms to a mosaic dataset. While adding data, the image processing will be defined based on user specified raster types. The processes normally include image georeferencing, orthorectification, pan-sharpening, image enhancement, etc. Additional processes such as color correction and seamline generation can be performed on the mosaic dataset to create seamless, color-balanced mosaics. The following geoprocessing model (Figure 8) shows the steps to create three types of ortho image products: first, a mosaic dataset that provides a dynamic image mosaic; second, an orthorectified mosaic stored in a well-known format such as a TIFF file, which can be used in any software package, and third, a folder of ortho image tiles that is based on a user defined tile size. 


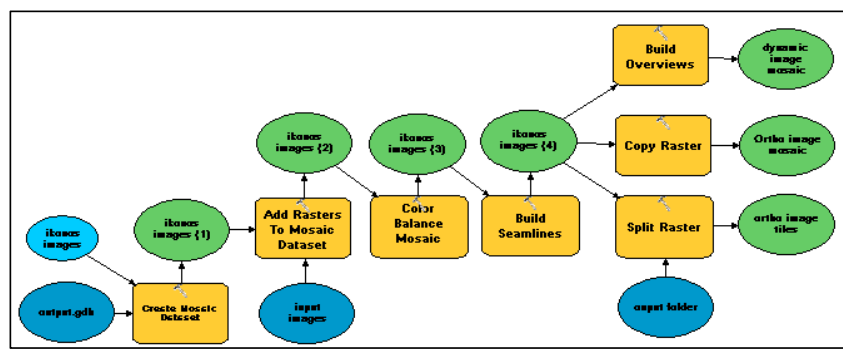

Figure 8. Geoprocessing model creating a virtual mosaic, a mosaicked TIFF, and image tiles

2) Cataloging a large collection of images: As the mosaic dataset manages the footprints in geodatabase but references the image data on disk, it becomes an advanced model for managing large image collections. First, a mosaic dataset of a large image collection can be created quickly as it does not load pixels into the geodatabase. Second, by taking advantage of the geodatabase technology, there is virtually no limit on the number of images that can be managed by a mosaic dataset. The limit is only in the hardware resources. Third, the mosaic dataset can manage data of various sensor platforms. Figure 9, shows the steps to create a mosaic dataset from Landsat and QuickBird images.Last, since the mosaic dataset footprint table contains information about the image, such as location, sensor type, image acquisition date, cloud cover, and so on, you can search and retrieve images from the mosaic dataset using attribute and location queries. A typical example of the search can be "all images acquired after the year 2000 with cloud cover less than 3\% and within my study area delineated by a polygon".

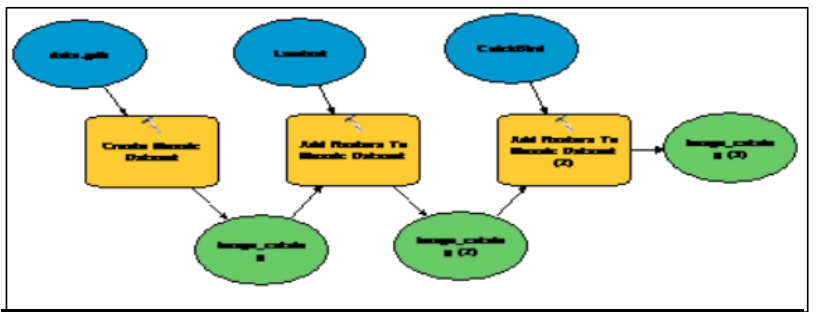

Figure 9. Geoprocessing model creating a mosaic dataset for cataloging and searching

3) Serving an image mosaic and catalog for internet access: Using the ArcGIS Server technology, the mosaic dataset can be served as an image service to provide dynamic mosaicking and cataloging capabilities. Figure 10, shows the search results from a Landsat service of the world using "AcquisitionDate $>$ date '2008-01 -01'” plus a polygon around the Great Lakes area in North America. Furthermore, once selected, users can directly use the services with only the specified images being processed, export the orthorectified images in a well-known image format, or download the original image with metadata information—straight from the server to the desktop machine or browser. Users can also export the mosaic to a well-known image format with a user expected projection.

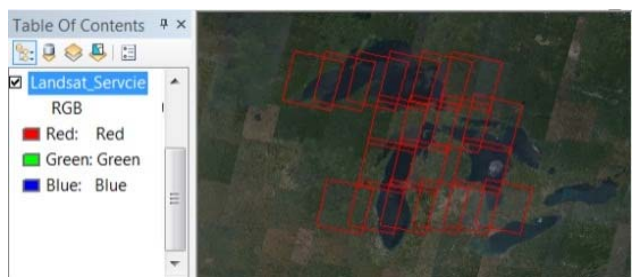

Figure 10. Search result from an image service served by a mosaic dataset

\subsubsection{Reference mosaic datasets}

Because of the nature of on-demand processing, multiple mosaic datasets can be created from the same source data by simply referencing the source mosaic dataset and adding raster functions. For example (Figure 11), from a multiple band mosaic dataset of Landsat image data, we can create a false color mosaic dataset by simply creating a reference mosaic dataset and adding a function to extract band, or create an NDVI mosaic dataset by adding a NDVI function.
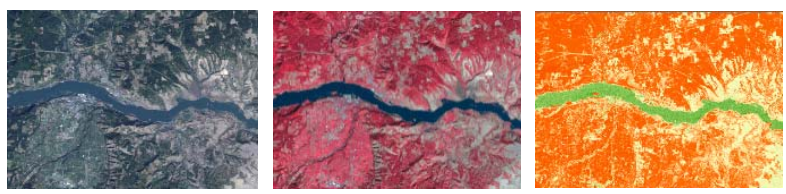

Figure 11. Mosaic dataset of RGB, False Color, and NDVI that reference the same source data

\subsubsection{Mosaic dataset updating}

Mosaic datasets provide an automatic updating mechanism. New images in the data folder can be automatically added to the mosaic dataset using the same raster type used in the mosaic dataset through a synchronize operation. Existing images in the mosaic dataset can also be updated if the sources or image properties are changed.

\subsubsection{Mosaic dataset cascading}

You can create a master mosaic dataset from one or multiple child mosaic datasets by using a special raster type called Table. This raster type will copy all the records and the raster type information from the child mosaic dataset to the master mosaic data. Any updates to the child mosaic datasets can be synchronized to the master mosaic dataset. This framework enables one to manage data in an organization that has multiple departments or teams. In the example showed in Figure 12, multiple counties create their own mosaic datasets to manage their

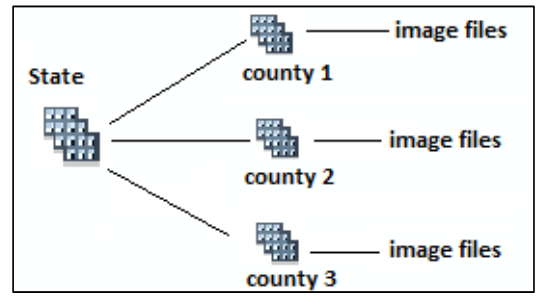

Figure 12. Cascaded mosaic dataset 
images within the county. The State can then create a master mosaic dataset by adding the mosaic datasets from each county to have a statewide mosaic dataset without having to duplicate the data. The State will then update the master mosaic dataset by synchronizing whatever changes that the counties have made to their child mosaic datasets (Figure 12).

The mosaic dataset can reduce the use of disk space, shorten the process time, and produce mosaics on demand based on a user's request. It is an advanced model in processing images, mosaicking images, cataloging images, and serving images.

\section{CONCLUSION}

ArcGIS's mosaic dataset and raster product data models have made the use of remote sensing images in GIS applications easier to GIS users. By using ArcGIS Server, a software product in the ArcGIS software family for serving GIS data, remote sensing images can be served easily and quickly to internet users. With the new data models, ArcGIS users can directly work with individual image scenes from many sensor platforms and organizations can manage and serve images within their organizations and through the internet. Furthermore, the new models can effectively reduce the cycle of building GIS databases, producing maps, and generating analysis results from remote sensing images. The integration of image processing and GIS has proved to be a successful solution for both GIS disaster applications and managing large collections of remote sensing images.

\section{ACKNOWLEGEMENTS}

The authors would like to thank Melanie Harlow from Esri for reviewing and editing the paper. 